\title{
Early short-term versus prolonged low-dose methylprednisolone therapy in acute lung
} injury

\author{
P.L. Silva*, C.S.N.B. Garcia*, P.A. Maronas*, V.R. Cagido\#, E.M. Negri", \\ N.R. Damaceno-Rodrigues", G.M. Ventura ${ }^{+}$, P.T. Bozza ${ }^{+}$, W.A. Zin" ${ }^{\#}$, V.L. Capelozzi", \\ P. Pelosi ${ }^{\S}$ and P.R.M. Rocco*
}

ABSTRACT: The present study compared the effects of early short-term with prolonged low-dose corticosteroid therapy in acute lung injury (ALI).

In total, 120 BALB/C mice were randomly divided into five groups. In the control group, saline was intratracheally (i.t.) instilled. In the ALI group, mice received Escherichia coli lipopolysaccharide (10 $\mu \mathrm{g}$ i.t.). ALI animals were further randomised into four subgroups to receive saline $\left(0.1 \mathrm{~mL}\right.$ i.v.) or methylprednisolone $\left(2 \mathrm{mg} \cdot \mathrm{kg}^{-1}\right.$ i.v. $)$ at $6 \mathrm{~h}, 24 \mathrm{~h}$ or daily (for 7 days, beginning at day 1). At 1, 3 and 8 weeks, in vivo and in vitro lung mechanics and histology (light and electron microscopy), collagen and elastic fibre content, cytokines in bronchoalveolar lavage fluid and the expression of matrix metalloproteinase (MMP)-9 and -2 were measured.

In vivo (static elastance and viscoelastic pressure) and in vitro (tissue elastance and resistance) lung mechanics, alveolar collapse, cell infiltration, collagen and elastic fibre content and the expression of MMP-9 and MMP-2 were increased in ALI at 1 week. Methylprednisolone led to a complete resolution of lung mechanics, avoided fibroelastogenesis and the increase in the expression of MMP-9 and MMP-2 independent of steroid treatment design.

Thus, early short-term, low-dose methylprednisolone is as effective as prolonged therapy in acute lung injury.

KEYWORDS: Acute lung injury, collagen, corticosteroid, elastance, lung histology, matrix metalloproteinase

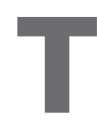
he use of corticosteroids in the treatment of acute lung injury (ALI) and acute respiratory distress syndrome (ARDS) has been the subject of great controversy and debate [1]. Corticosteroid therapy in ALI/ARDS has been studied mainly in: 1) prevention of ALI/ARDS in high-risk patients [2]; 2) early treatment with high-dose, short-time therapy [3]; and 3) prolonged therapy with low dose, in unresolved cases [4].

Recently, some clinical studies have shown beneficial effects when corticosteroid therapy was begun at the early phase of ALI/ARDS at low dose [5-7]. Along these lines, it was demonstrated that a single low dose of methylprednisolone in the early phase of ALI was effective at inhibiting the remodelling process of lung parenchyma [8]. Additionally, the early beneficial effects of corticosteroid on extracellular matrix
(ECM) remained unaltered 30 days after the induction of lung injury [8]. Therefore, if an early single dose of corticosteroid was able to adequately regulate the remodelling process, extended steroid therapy may not be necessary, minimising the side-effects related to its prolonged use. However, other authors described that, to achieve disease resolution, it is important to prolong corticosteroid treatment for as long as necessary [1, 7].

In the present study, the use of short-term lowdose corticosteroid was compared with prolonged therapy in the early phase of ALI. For this purpose, in vivo and in vitro respiratory mechanics, lung histology, inflammatory response and the structural remodelling of lung parenchyma were analysed in a murine model of ALI, 1, 3 and 8 weeks after the induction of lung injury.
AFFILIATIONS

*Laboratory of Pulmonary Investigation, \#Laboratory of Respiration Physiology, Carlos Chagas Filho Biophysics Institute, Federal University of Rio de Janeiro,

+Laboratory of Immunopharmacology, Oswaldo Cruz Institute, FIOCRUZ, Rio de Janeiro,

'Dept of Pathology, Faculty of Medicine, University of São Paulo, São Paulo, Brazil, and

${ }^{\S}$ Dept of Ambient, Health and Safety, University of Insubria, Varese, Italy.

\section{CORRESPONDENCE}

P.R.M. Rocco

Laboratory of Pulmonary Investigation, Carlos Chagas Filho Biophysics Institute, Federal University of Rio de Janeiro, Centro de Ciências da Saúde, Avenida Carlos Chagas Filho, s/n, Bloco G-014, Ilha do Fundão, 21941902, Rio de Janeiro, RJ, Brazil Fax: 552122808193

E-mail:prmrocco@biof.ufr..br

Received:

April 052008

Accepted after revision:

September 272008

SUPPORT STATEMENT

This study received financial support from the Centres of Excellence

Program (Rio de Janeiro, Brazil), the Brazilian Council for Scientific and Technological Development, the Carlos Chagas Filho Rio de Janeiro State Research Supporting

Foundation, and the São Paulo State Research Support Foundation (Brazil).

STATEMENT OF INTEREST

None declared. 


\section{METHODS}

The present study was approved by the Ethics Committee of the Carlos Chagas Filho Institute of Biophysics, Health Sciences Centre, Federal University of Rio de Janeiro (Rio de Janeiro, Brazil). All animals received humane care in compliance with the Principles of Laboratory Animal Care formulated by the National Society for Medical Research (Bethesda, MD, USA) and the Guide for the Care and Use of Laboratory Animals prepared by the National Academy of Sciences (Bethesda).

\section{Study design}

A total of $120 \mathrm{BALB} / \mathrm{c}$ mice (20-25 g) were randomly divided into five groups. In the control group $(\mathrm{n}=18), 0.05 \mathrm{~mL}$ saline $(0.9 \% \mathrm{NaCl})$ was intratracheally (i.t.) instilled. In the ALI group, mice received Escherichia coli lipopolysaccharide (LPS; serotype O55:B5) at $10 \mu \mathrm{g}$ in $0.05 \mathrm{~mL}$ of saline per mouse, i.t. For i.t. instillation, mice were anaesthetised with sevoflurane, a 1-cm midline cervical incision was made to expose the trachea, and LPS or saline were instilled with a bent 27-gauge tuberculin needle. The cervical incision was closed with 5.0 silk suture and mice returned to their cage, where they recovered rapidly after surgery. ALI animals were further randomised into four subgroups (18 mice in each) to receive saline $\left(0.1 \mathrm{~mL}\right.$ i.v.) or methylprednisolone $\left(2 \mathrm{mg} \cdot \mathrm{kg}^{-1}\right.$ diluted in $0.1 \mathrm{~mL}$ of saline injected through the tail vein) at $6 \mathrm{~h}, 24 \mathrm{~h}$ or daily (for 7 days, beginning at day 1).

All five groups were studied at 1, 3 and 8 weeks $(n=6$ at each time point), when the animals were sedated (with diazepam, $1 \mathrm{mg}$ i.p.), anaesthetised (with pentobarbital sodium, $20 \mathrm{mg} \cdot \mathrm{kg}^{-1}$ i.p.), tracheotomised, paralysed (with vecuronium bromide, $0.005 \mathrm{mg} \cdot \mathrm{kg}^{-1}$ i.v.), and ventilated with a constant flow ventilator (Samay VR15; Universidad de la Republica, Montevideo, Uruguay) with the following parameters: frequency of 100 breaths $\cdot \mathrm{min}^{-1}$, tidal volume $(V \mathrm{~T})$ of $0.2 \mathrm{~mL}$ and fraction of inspired oxygen of 0.21 . Subsequently, the chest wall was surgically removed and a positive end-expiratory pressure of $2 \mathrm{cmH}_{2} \mathrm{O}$ was applied. After a 10-min ventilation period, in vivo lung mechanics were computed and, at the end of the experiments (after $\sim 30 \mathrm{~min}$ ), lungs were removed and prepared for in vitro lung mechanical analysis and histology.

\section{Mechanical parameters}

Airflow and tracheal pressure $(P \operatorname{tr})$ were measured. $V \mathrm{~T}$ was calculated by digital integration of flow signal. In vivo lung mechanics (static elastance $\left(E_{\mathrm{st}}, \mathrm{L}\right)$, resistive pressure $(\Delta P 1, \mathrm{~L})$ and viscoelastic/inhomogeneous pressure $(\Delta P 2, \mathrm{~L}))$ were measured by the end-inflation occlusion method [9]. In an open chest preparation, $P$ tr reflects transpulmonary pressure. Strips $(2 \times 2 \times 10 \mathrm{~mm})$ were then cut from the periphery of each left lung in all groups, suspended vertically in a Krebs-Henseleith organ bath continuously bubbled with $95 \% \mathrm{O}_{2} / 5 \% \mathrm{CO}_{2}$ and maintained at $37^{\circ} \mathrm{C}$ [10]. In vitro lung mechanics (tissue resistance $(R)$, elastance $(E)$, and hysteresivity $(\eta)$ ) were calculated from the oscillatory recordings [11].

\section{Histology and immunohistochemistry}

Light microscopy

The right lungs were quick-frozen by immersion in liquid nitrogen and fixed with Carnoy's solution [10]. Slices $4 \mu \mathrm{m}$ thick were cut and stained with haematoxylin and eosin.
Morphometric analysis was performed using an integrated eyepiece with a coherent system consisting of a 100-point and 50-line grid (known length) coupled to a conventional light microscope (Axioplan; Zeiss, Oberkochen, Germany). The volume fraction of the lung occupied by hyperinflated structures (alveolar ducts, alveolar sacs or alveoli wider than $120 \mu \mathrm{m}$ ), collapsed alveoli (alveoli with rough or plicate walls) or normal pulmonary areas, and the amount of polymorphoand mononuclear cells and pulmonary tissue were determined by the point-counting technique [12]. Specific staining methods to quantify collagen and elastic fibre content in the alveolar septa were also used [8, 10]. In each mouse, 20 different microscopic fields were randomly selected to quantify the amount of collagen and elastic fibres by a digital image analysis system and specific software (Bioscan-Optimas 5:1; Bioscan, Edmond, WA, USA) under $200 \times$ magnification. The images were generated by a microscope (Axioplan) connected to a camera (Sony Trinitron CCD; Sony, Tokyo, Japan) and fed into a computer through a frame grabber (Oculus TCX; Coreco, St Laurent, QC, Canada) for offline processing. The thresholds for collagen and elastic fibres were established after enhancement of contrast up to the point where the fibres were easily identified as either birefrigent (collagen) or black (elastic) bands. Bronchi and blood vessels were carefully avoided during the measurements. The area occupied by the collagen and elastic fibres in each alveolar septum was divided by the septum length to eliminate any bias due to septal oedema or alveolar collapse. The results were expressed as the amount of collagen or elastic fibres per unit of septal length $\left(\mu \mathrm{m}^{2} \cdot \mu \mathrm{m}^{-1}\right)[10]$.

\section{Transmission electron microscopy}

Three slices $(2 \times 2 \times 2 \mathrm{~mm})$ were cut from three different segments of the left lung to obtain a stratified random sample. A specimen was then fixed in $2.5 \%$ glutaraldehyde and phosphate buffer. Ultrathin sections were investigated using a transmission electron microscope (JEOL 1010 Transmission Electron Microscope; JEOL, Tokyo, Japan). For each electron microscopy image (15 per animal) an injury score was determined. The following parameters were analysed: alveolar capillary barrier, type II epithelial cell, type I epithelial cell, collagen fibre and endothelial cell, according to a 5-point semiquantitative severity-based scoring system. The pathological findings were graded as follows: 0) normal lung parenchyma; 1) changes in $1-25 \%$; 2) changes in $26-50 \%$; 3 ) changes in $51-75 \%$; and 4 ) changes in $76-100 \%$ of the examined tissue.

\section{Immunohistochemistry}

Strips $(2 \times 2 \times 10 \mathrm{~mm})$ from the right lung were fixed with $4 \%$ paraformaldehyde and embedded in paraffin for immunohistochemical study. Matrix metalloproteinase (MMP)-9 and -2 were identified by immunohistochemical staining with the peroxidise-LSAB+ technique (Dako, Carpinteria, CA, USA). Antigen retrieval was carried out by pressure-cooking the slides for $3 \mathrm{~min}$ in $10 \mathrm{mM}$ citric acid buffer at $\mathrm{pH} \mathrm{6.0,} \mathrm{and}$ endogenous peroxidase activity was blocked by placing the slides in $2 \%$ hydrogen peroxide for $30 \mathrm{~min}$. Slides were then rinsed in deionised water followed by Tris-buffered saline containing $0.1 \%$ bovine serum albumin, incubated in $20 \%$ normal rabbit serum for $10 \mathrm{~min}$, and then incubated with 
primary antibody overnight at $4{ }^{\circ} \mathrm{C}$ (MMP-9 mouse monoclonal 56-2A4 (Dako) at 1:100 dilution and MMP-2 mouse monoclonal (clone VC2; NeoMarkers, Fremont, CA, USA) at 1:1000 dilution). This was followed by streptavidin combined in vitro with biotinylated horseradish peroxidase (Dako) at a dilution of 1:1000. The reaction product was developed using diaminobenzidine tetrahydrochloride. Finally, the slides were counterstained with haematoxylin, dehydrated and mounted in resinous mountant. Known positive controls were included in each run, as well as negative controls without the primary antibody [13]. Any brown cytoplasmic staining of cells (macrophages, neutrophils and alveolar epithelial cells) characterised positive expression for MMP-9 and MMP-2, and morphometry and an eyepiece grid with 100 points and 50 lines were used to quantify the staining as follows. First, at $100 \times$ magnification, the region of highest expression was selected. Secondly, the magnification was increased to $400 \times$ in order to count the fraction of points overlaying positively stained structures. The average of 10 microscopic fields provided the final result as a percentage of stained structures.

\section{Evaluation of bronchoalveolar lavage fluid}

The remaining 30 animals (six mice per group) were submitted to the same protocol as previously described for obtaining aliquots of bronchoalveolar lavage fluid (BALF) [14, 15]. Amounts of interleukin (IL)-6 and keratinocyte chemoattractant (KC) were quantified by ELISA on cell-free BALF according to manufacturer's protocol (Duo set; R\&D Systems, Minneapolis, MN, USA).

\section{Statistical analysis}

Data are presented as mean \pm SEM or median (25th to 75 th percentiles) as appropriate. Comparisons between the experimental groups were performed using one-way ANOVA or the Kruskal-Wallis test, followed by post hoc Tukey's test, depending on data distribution. Fisher's exact test was used to compare the mortality rates among the different weeks. The relationships between mechanical parameters and the amount of collagen and elastic fibres were evaluated by Spearman's correlation. In all tests the significance level was set at $5 \%$.

\section{RESULTS}

The mortality rate of ALI mice was significantly lower at 1 and 3 weeks compared with 8 weeks (15 versus $40 \%$, respectively; $\mathrm{p}<0.001)$. Methylprednisolone led to no deaths independent of the corticosteroid treatment design.

In vivo (Est, $\mathrm{L}, \Delta P 1, \mathrm{~L}$ and $\Delta P 2, \mathrm{~L}$; fig. 1 ) and in vitro ( $E$ and $R$; fig. 2) lung mechanics were more markedly affected in ALI than in control mice at 1 week (fig. 1). Est,L returned to control values at 8 weeks, but $\Delta P 2, \mathrm{~L}, E$ and $R$ remained elevated (figs 1 and 2). Methylprednisolone led to a complete resolution of in vivo and in vitro lung mechanics. $\eta$ was similar in all groups (fig. 2).

Typical photomicrographs of lung parenchyma from control and ALI groups treated with saline or methylprednisolone at $6 \mathrm{~h}, 24 \mathrm{~h}$ or daily are shown in figure 3 . Since the lung histology of control groups was similar at 1, 3 and 8 weeks, only one time-point is depicted (1 week). ALI groups treated with saline presented lung histological changes, including atelectasis, interstitial oedema and cell infiltration (fig. 3).
Although less intense at 8 weeks, these changes remained more prominent than in the control groups. All ALI groups treated with methylprednisolone showed less interstitial oedema and neutrophilic infiltration, independent of time (fig. 3).

Morphometric examination of lungs also demonstrated significant increase in alveolar collapse and the number of polymorphonuclear cells in ALI groups compared with control at all time-points (table 1). However, in ALI groups, the fractional area of alveolar collapse and the number of polymorphonuclear cells were lower at 8 weeks compared with 1 and 3 weeks. Data from 1 and 3 weeks did not differ from each other. Mononuclear cell content was smaller in ALI groups compared with control (table 1). Methylprednisolone reduced the fractional area of alveolar collapse in ALI groups, but remained higher than control at 1, 3 and 8 weeks. Alveolar hyperinflation was not observed in any group (table 1 ).
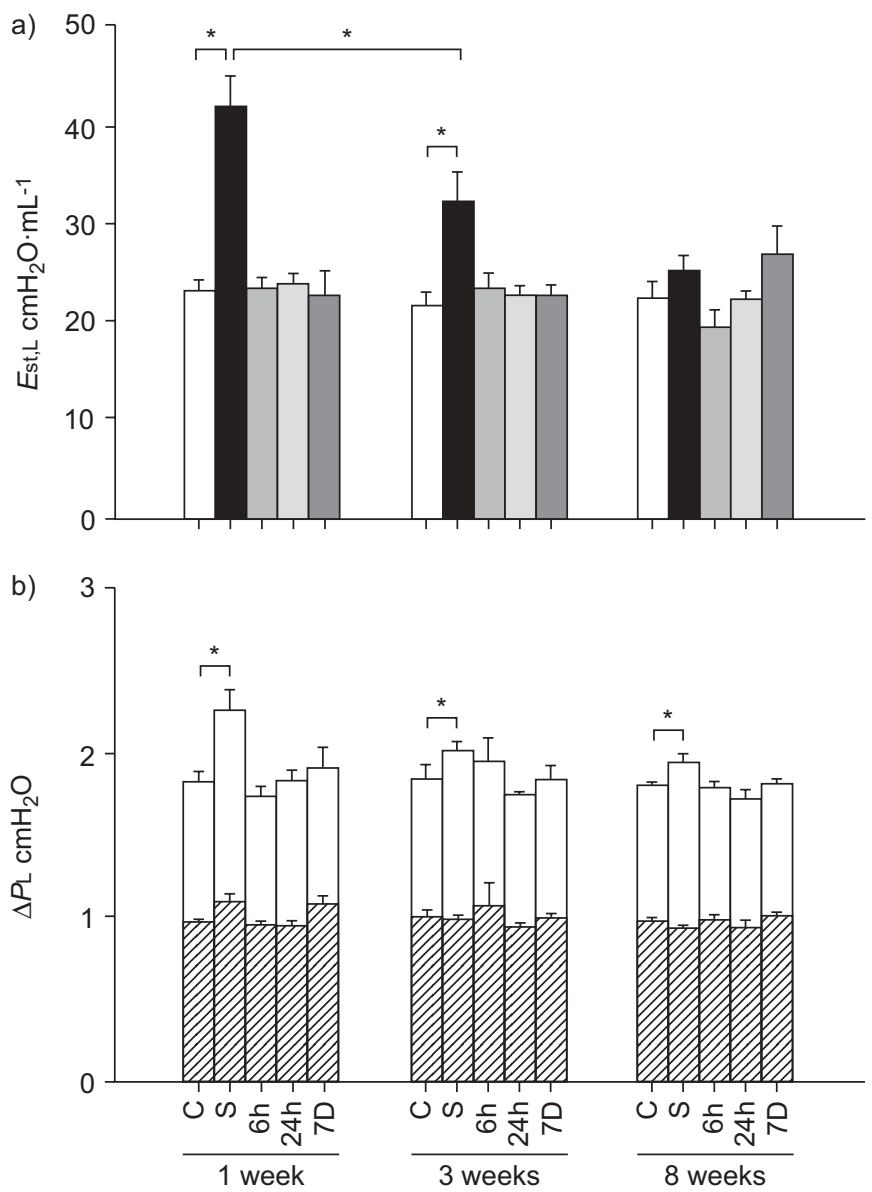

FIGURE 1. a) Lung static elastance (Est,L) and b) stacked bar chart plot data In the control group $(C)$, saline was intratracheally instilled. In the acute lung injury (ALI) groups, mice received Escherichia coli lipopolysaccharide. The ALI groups were treated with saline (S) or methylprednisolone $\left(2 \mathrm{mg} \cdot \mathrm{kg}^{-1} \mathrm{i} . \mathrm{v}\right.$.) at $6 \mathrm{~h}, 24 \mathrm{~h}$ or daily (for 7 days (7D), beginning at day 1 ). All groups were analysed at 1,3 and 8 weeks. $\triangle P L$ : lung pressures, either resistive pressure $(\mathbb{Z})$ or viscoelastic/ inhomogeneous pressure $(\square)$ of the lung. Data are presented as mean \pm SEM. * $p<0.05$. 
Collagen and elastic fibre content in the alveolar septa were significantly higher in ALI compared with the control group at 1, 3 and 8 weeks (fig. 4). Methylprednisolone avoided the increment in collagen and elastic fibre content, independent of the treatment scheme and the time course of ALI.

Figure 5 shows the time course of ultramicroscopic changes in lung parenchyma. In the control group, alveolar spaces and epithelial cells were preserved (fig. 5). At week 1, the ALI group showed cytoplasmatic degeneration of type II pneumocytes, and thickening of the interstitium due to an increase of ECM elements, such as collagen fibres (fig. 5). At week 3, electron microscopy showed intra-alveolar proliferation of myofibroblasts with obliterative fibrosis, and proliferation of type II epithelial cells. At this time, there was an increased number of type I collagen fibres. At week 8 , there was a replication of type II epithelial cells, repopulating the denuded air-lung interface, and myofibroblast proliferation determined new alveolar septa formation, with a predominant increase of type I collagen fibres (fig. 5). Corticosteroid therapy decreased the number of fibroblasts, type I and III collagen fibres and elastin (fig. 6; table 2).

Considering all groups, in vitro mechanics ( $E$ and $R$ ) were significantly correlated with total cell count $(\mathrm{p}=0.007(\mathrm{r}=0.37)$ and $\mathrm{p}=0.001 \quad(\mathrm{r}=0.44)$, respectively $)$, collagen $(\mathrm{p}<0.001$ $(r=0.65)$ and $p<0.001(r=0.57)$, respectively) and elastic fibre content $(\mathrm{p}=0.01(\mathrm{r}=0.36)$ and $\mathrm{p}=0.01(\mathrm{r}=0.34)$, respectively).

The levels of IL- 6 and KC at 1 week in control, ALI and methylprednisolone-treated animals were similar among the groups (mean \pm SEM IL-6: control 0.005 \pm 0.001 , ALI $0.007 \pm 0.003$, methylprednisolone $6 \mathrm{~h} \quad 0.004 \pm 0.002,24 \mathrm{~h}$ $0.008 \pm 0.002$ and 7 days $0.005 \pm 0.003 \mathrm{ng} \cdot \mathrm{mL}^{-1} ; \mathrm{KC}$ : control $0.30 \pm 0.01$, ALI $0.32 \pm 0.02$, methylprednisolone 6 h $0.12 \pm 0.02$, $24 \mathrm{~h} 0.16 \pm 0.03$ and 7 days $0.21 \pm 0.05 \mathrm{ng} \cdot \mathrm{mL}^{-1}$ ).

The number of cells expressing MMP-9 and -2 was higher in ALI than in the control group, independent of the time-point. Macrophages expressed more MMP-9 than neutrophils at 1, 3 and 8 weeks. The expression of MMP- 9 was reduced with the time course of ALI. Methylprednisolone diminished MMP-9 expression in both neutrophils and macrophages (figs 7 and 8). Weak expression of MMP-2 was detected in alveolar macrophages, alveolar lining epithelium, alveolar septal interstitium and interstitial cells in the lung of both control and treated animals. MMP-2 expression in alveolar macrophages increased significantly in ALI groups at 1, 3 and 8 weeks compared with control (table 3; fig. 8).

\section{DISCUSSION}

In the present study, it was observed that early short-term, low-dose methylprednisolone was as effective as prolonged therapy in ALI, inhibiting in vivo and in vitro lung mechanical changes, fibroelastogenesis and MMP-9 and MMP-2 expression, while minimising the amount of atelectasis and tissue cellularity.

ALI was induced by i.t. instillation of E. coli LPS. LPS produces a well-characterised model of ALI, mimicking morphological and functional changes observed in clinical situations [13-15]. At present, there is no established treatment to halt the progression of ALI. The ideal pharmacological treatment
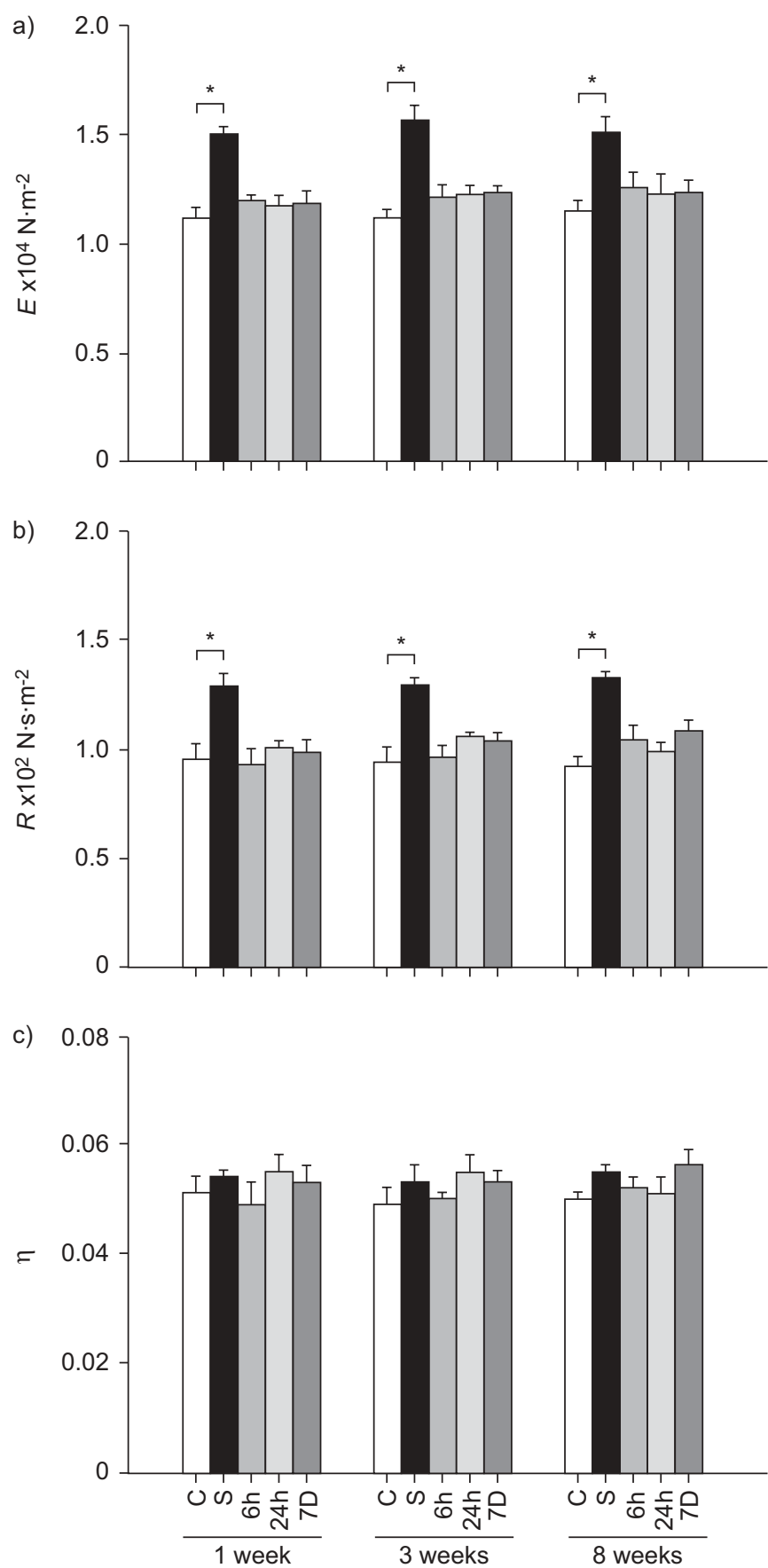

FIGURE 2. a) Tissue elastance $(E)$, b) resistance $(R)$ and c) hysteresivity $(\eta)$ at $1 \mathrm{~Hz}$. In the control (C) group, saline was intratracheally instilled. In the acute lung injury (ALI) groups, mice received Escherichia coli lipopolysaccharide. The ALI groups were treated with saline $(\mathrm{S})$ or methylprednisolone $\left(2 \mathrm{mg} \cdot \mathrm{kg}^{-1}\right.$ i.v. $)$ at $6 \mathrm{~h}$, $24 \mathrm{~h}$ or daily (for 7 days (7D), beginning at day 1 ). All groups were analysed at 1, 3 and 8 weeks. Data are presented as mean \pm SEM. ${ }^{*}: p<0.05$.

should promote epithelial and endothelial cell repair, stop the progress of fibroproliferation and decrease ECM deposition while stimulating its net reabsorption. Corticosteroids may have a broad range of effects on the host's defence response, inhibiting nuclear factor- $\mathrm{\kappa B}$ and activator protein-1, as well as blocking the transcription of several cytokines relevant in ALI/ ARDS [16, 17]. 

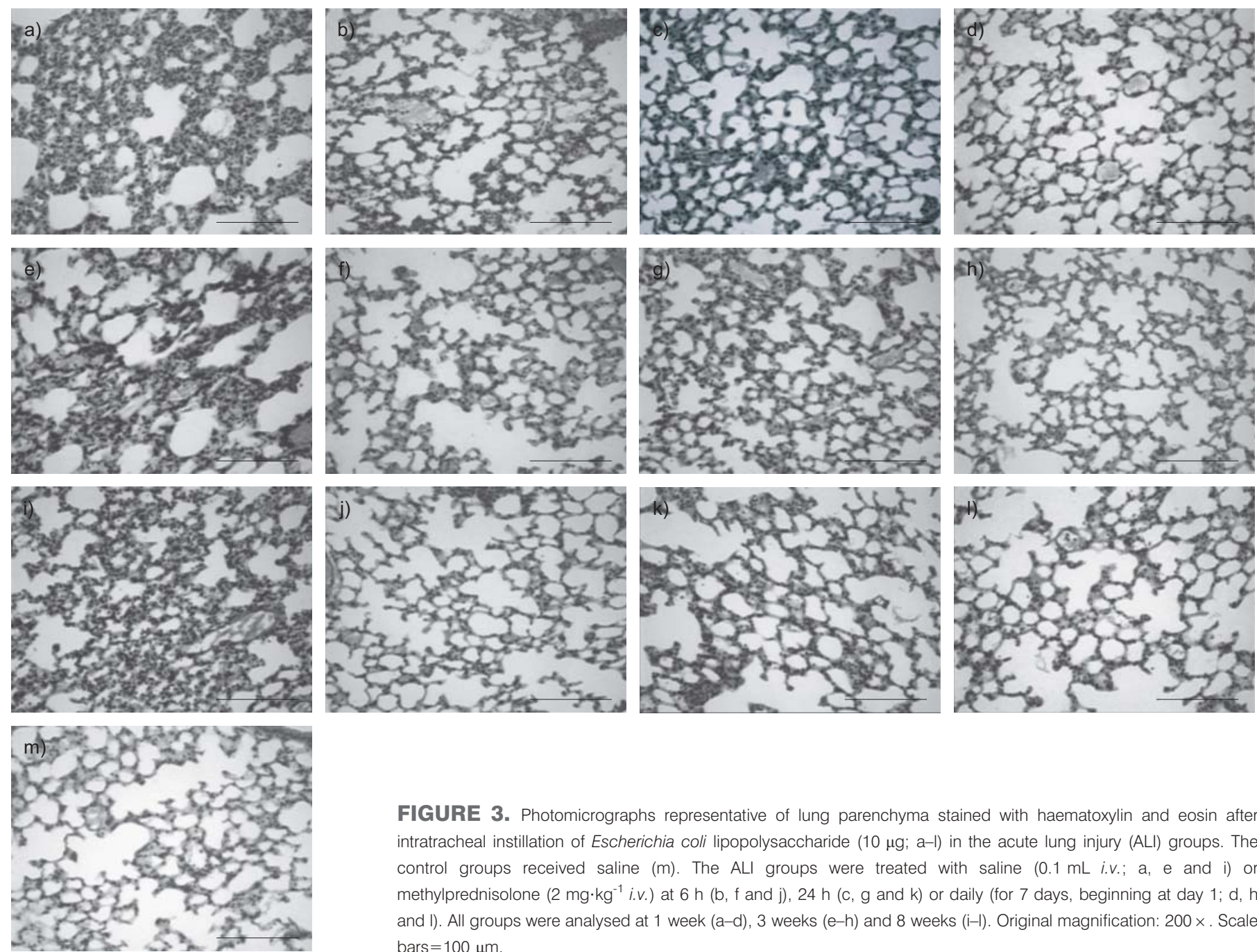

FIGURE 3. Photomicrographs representative of lung parenchyma stained with haematoxylin and eosin after intratracheal instillation of Escherichia coli lipopolysaccharide (10 $\mu \mathrm{g}$; a-l) in the acute lung injury (ALI) groups. The control groups received saline $(\mathrm{m})$. The ALI groups were treated with saline $(0.1 \mathrm{~mL}$ i.v.; a, e and i) or methylprednisolone ( $2 \mathrm{mg} \cdot \mathrm{kg}^{-1}$ i.v.) at $6 \mathrm{~h}(\mathrm{~b}, \mathrm{f}$ and j), $24 \mathrm{~h}$ (c, g and $\mathrm{k}$ ) or daily (for 7 days, beginning at day $1 ; \mathrm{d}, \mathrm{h}$ and I). All groups were analysed at 1 week (a-d), 3 weeks (e-h) and 8 weeks (i-l). Original magnification: $200 \times$. Scale bars $=100 \mu \mathrm{m}$.

In the ALI group, Est,L was altered at week 1 and improved progressively with the time course of the disease, returning to normal values 8 weeks after the induction of lung injury (fig. 1). Conversely, $\Delta P 1, \mathrm{~L}$ and $\Delta P 2, \mathrm{~L}$ remained elevated in the ALI group at 1,3 and 8 weeks. These changes in $\Delta P 1, \mathrm{~L}$ could be attributed to the release of endogenous mediators following endotoxemia, yielding active airway constriction [18]. The increase in $\Delta P 2, \mathrm{~L}$ could reflect the presence of alveolar collapse, distortion of patent alveoli, oedema and inflammation with neutrophils (table 1; figs 3, 5 and 6), as well as changes in ECM components (fig. 4) characterised by fibroelastosis [8, 10, 13]. Since the animals used in the current study were spontaneously breathing, the present data suggest that fibroelastosis may be partially independent of the effects of mechanical ventilation, but directly related to the physiological evolution of ALI/ARDS.

Besides being the most common corticosteroid used in clinical trials $[3,7,19]$, methylprednisolone was chosen because it has better concentration in the lungs than other corticosteroids due to its larger volume of distribution, longer mean residence time and greater retention in the epithelial lining fluid of the alveoli [20]. A relatively low dose of methylprednisolone was employed $\left(2 \mathrm{mg} \cdot \mathrm{kg}^{-1}\right.$ i.v.), since this had been proved to be effective at avoiding lung mechanical changes in mild ALI and minimising alterations in tissue impedance and ECM components in severe ALI [8]. Few studies have investigated the effects of low-dose corticosteroids in early ALI/ARDS. In clinical studies, LEE et al. [6] observed that the early use of a low dose of methylprednisolone in ARDS was associated with a reduced mortality rate after thoracic surgery. Furthermore, corticosteroids inhibited systemic inflammation, preventing overwhelming compensatory anti-inflammatory responses [21]. Confalonieri et al. [22] reported beneficial effects of low-dose hydrocortisone on lung function and mortality in community-acquired pneumonia. Recently, MEDURI et al. [7] reported beneficial effects of low-dose methylprednisolone in a randomised controlled trial in early ARDS, showing a significant improvement in the lung injury score and a reduction in systemic inflammatory markers in comparison with controls. In contrast, a large multicentre randomised trial showed no benefits on mortality when low-dose methylprednisolone was given in late ALI/ARDS [19]. There is a wide window, which may impair the corticosteroid effects, since the fibroproliferative phase begins early in the course of lung injury [23]. Additionally, prolonged corticosteroid treatment in 
TABLE 1 Morphometric parameters and cellularity in lung parenchyma

\begin{tabular}{|c|c|c|c|c|c|}
\hline \multicolumn{6}{|l|}{1 week } \\
\hline ALI+saline & $63.7 \pm 2.4^{*}$ & $36.3 \pm 2.3^{*}$ & $48.5 \pm 2.9^{*}$ & $8.2 \pm 1.8^{*}$ & $56.7 \pm 3.3^{*}$ \\
\hline M-6h & $78.3 \pm 0.6^{\star . \#}$ & $21.7 \pm 0.6^{\star, \#}$ & $9.3 \pm 0.4^{* . \#}$ & $16.6 \pm 0.4$ & $26.0 \pm 1.7^{*, \#}$ \\
\hline$M-24 h$ & $75.8 \pm 2.1^{* . \#}$ & $24.2 \pm 2.1^{\star * \#}$ & $18.8 \pm 0.7^{\star . \#}$ & $16.3 \pm 1.4$ & $35.1 \pm 5.8^{*, \#}$ \\
\hline Control & $92.4 \pm 1.5$ & $7.6 \pm 1.5$ & $2.9 \pm 0.7$ & $14.5 \pm 1.0$ & $17.4 \pm 0.8$ \\
\hline ALI+saline & $64.2 \pm 3.2^{\star}$ & $35.8 \pm 3.0^{*}$ & $45.1 \pm 2.9^{*}$ & $7.0 \pm 1.6^{*}$ & $52.0 \pm 4.2^{*}$ \\
\hline M-6h & $75.7 \pm 1.3^{\star, \#}$ & $24.2 \pm 1.3^{\star, \#}$ & $8.4 \pm 0.5^{\star, \#}$ & $14.0 \pm 1.0$ & $22.4 \pm 1.4^{*}$ \\
\hline M-24h & $75.5 \pm 2.3^{\star . \#}$ & $24.5 \pm 2.30^{* . \#}$ & $17.3 \pm 0.6^{\star . \#}$ & $16.7 \pm 2.1$ & $33.9 \pm 1.5^{*}$ \\
\hline$M-7 D$ & $76.4 \pm 1.6^{*, \#}$ & $23.6 \pm 1.4^{\star, \#}$ & $14.6 \pm 0.7^{*, \#}$ & $12.2 \pm 0.7$ & $26.9 \pm 0.6^{*}$ \\
\hline M-24h & $80.5 \pm 2.5^{*}$ & $19.5 \pm 2.3^{*}$ & $9.0 \pm 0.6^{\star, \#}$ & $10.9 \pm 1.4$ & $19.9 \pm 1.3^{*}$ \\
\hline$M-7 D$ & $80.4 \pm 0.8^{*}$ & $19.6 \pm 0.8^{*}$ & $10.0 \pm 1.6^{* . \#}$ & $8.1 \pm 0.7$ & $18.1 \pm 2.2^{*}$ \\
\hline
\end{tabular}

Data are presented as mean \pm SEM \%, with six lungs in each group and 10 random, non-coincident microscopic fields analysed in each lung. In the control group, saline was intratracheally instilled $(0.05 \mathrm{~mL}$ ), and the acute lung injury (ALI) mice received Escherichia coli lipopolysaccharide (10 $\mu \mathrm{g})$. The ALI groups were treated with saline (0.1 mL i.v.) or methylprednisolone ( $\mathrm{M} ; 2 \mathrm{mg} \cdot \mathrm{kg}^{-1}$ i.v.) at $6 \mathrm{~h}, 24 \mathrm{~h}$ or daily (for 7 days (7D), beginning at day 1). All groups were analysed at 1,3 and 8 weeks. PMN: polymorphonuclear cells; MN: mononuclear cells. *: $p<0.05$ compared with control; ${ }^{*}: p<0.05$ compared with ALI+saline at same time-point; ${ }^{\circ}: p<0.05$ compared with ALI+saline at 1 week.

conjunction with neuromuscular blocking agents may lead to prolonged neuromuscular weakness, delaying the weaning process [24].

In the current study, methylprednisolone, independent of the dosage regimen, normalised all lung mechanical parameters after 1 week. Furthermore, a single dose of corticosteroid, compared with repeated doses, was able to regulate the remodelling process adequately, suggesting that extended steroid therapy may not be necessary, which would minimise the side-effects related to prolonged use. This rapid effect could be explained by the normalisation of surfactant production and its tenso-active properties due to the corticosteroid [25], yielding an increase in lung aeration and a major reduction in atelectatic areas. The same occurred in relation to fibroelastosis, suggesting that early corticosteroid treatment
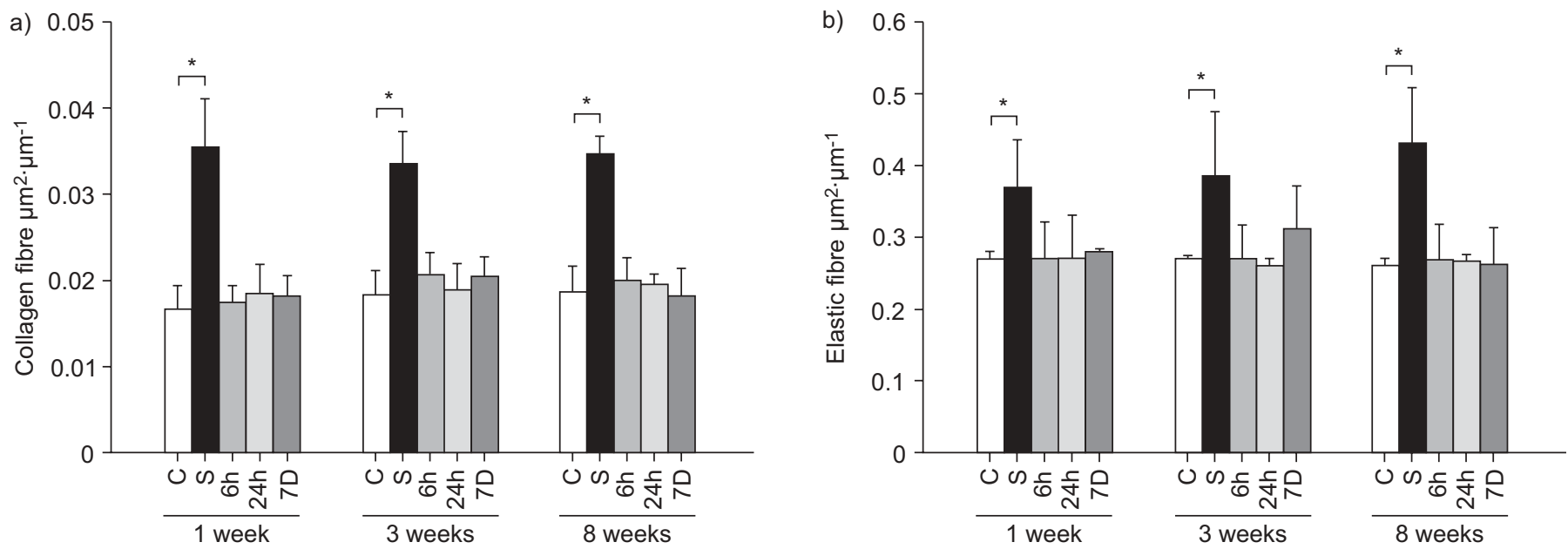

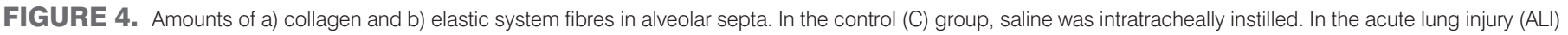

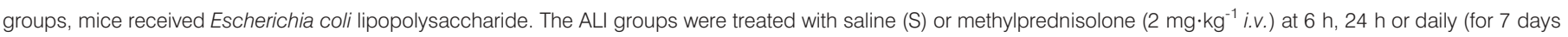
(7D), beginning at day 1). All groups were analysed at 1, 3 and 8 weeks. Data are presented as mean \pm SEM. ${ }^{*}: p<0.05$. 

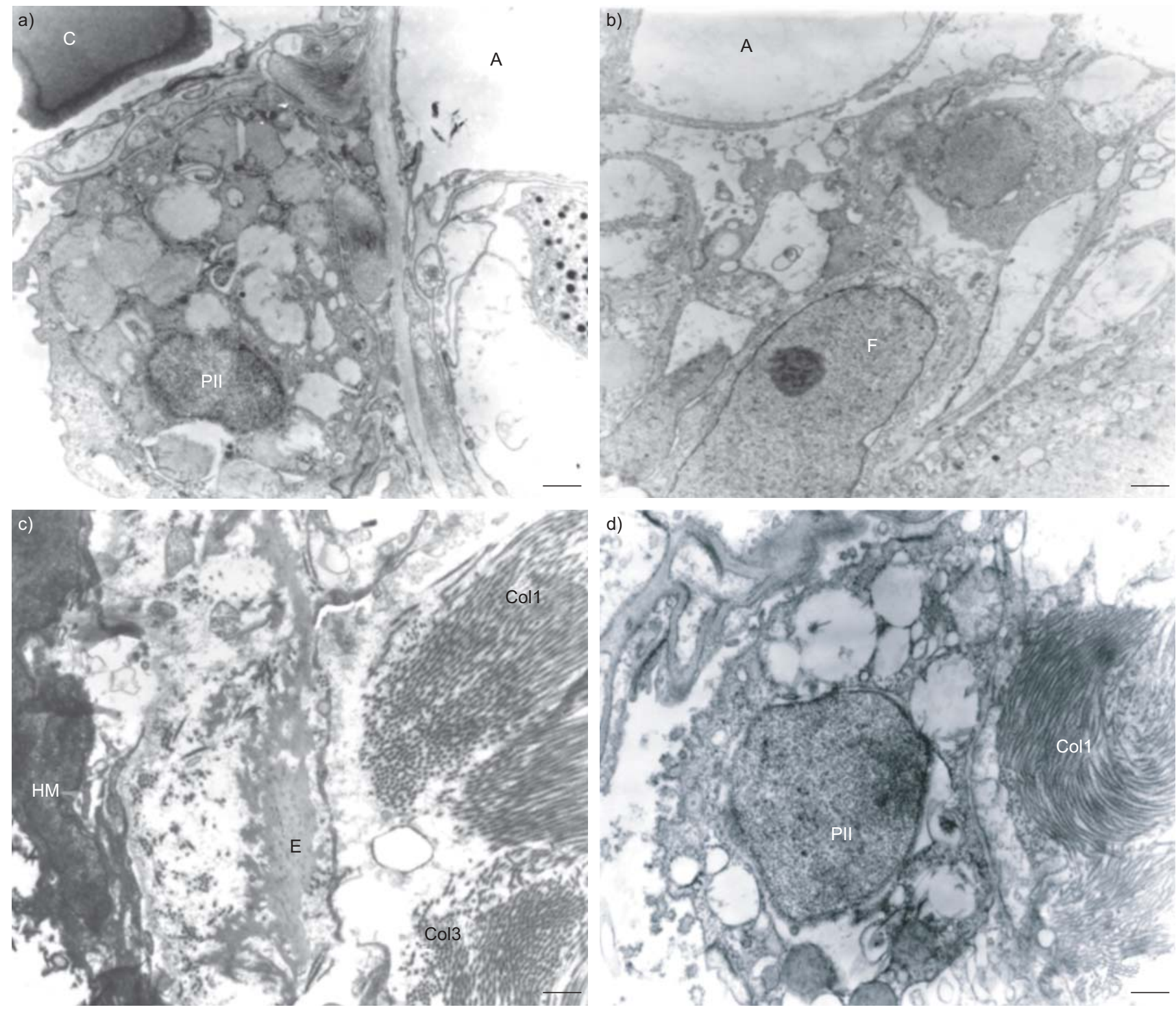

FIGURE 5. Electron microscopy of lung parenchyma after a) intratracheal instillation of saline as control, or b-d) Escherichia coli lipopolysaccharide (10 $\mu \mathrm{g})$ in acute lung injury (ALI) groups treated with saline ( $0.1 \mathrm{~mL}$ i.v.). ALI lungs were analysed at b) 1 week, c) 3 weeks and d) 8 weeks. a) Note the preserved type II pneumocytes (PII) and alveolar interstitial wall with normal components of the extracellular matrix. b) Note the presence of activated fibroblasts $(\mathrm{F})$ at 1 week. c) Hyaline membrane (HM) and fibroblasts were seen in the alveolar air barrier, as well as type III collagen fibres (Col3) and elastic fibres (E). d) At 8 weeks, only type I collagen fibres (Col1) and elastin were present. C: capillary; A: alveolar space. Scale bars $=500 \mathrm{~nm}$.

may prevent not only early but also late collagen and elastic fibre overproduction. Conversely, methylprednisolone only minimised the inflammatory response, as evaluated by polymorphonuclear cells and cellularity in the lung parenchyma. Therefore, ALI induced a long-term inflammatory reaction in the lung, which was limited but not abolished by methylprednisolone treatment.

Additionally, the current authors evaluated the levels of inflammatory cytokines (IL-6 and KC) during the time course of ALI (see online supplementary material). No significant changes in IL-6 and KC were observed in control, ALI and methylprednisolone-treated groups at 1,3 or 8 weeks.
However, a previous study showed that methylprednisolone therapy $6 \mathrm{~h}$ after ALI induction significantly inhibited the increase of IL-6 and KC at $24 \mathrm{~h}$ [15], suggesting that corticosteroid was effective in reducing the inflammatory response in the study animals. Nevertheless, since IL-6 and KC were measured after 1 week, it was not possible to observe any methylprednisolone effect on inflammatory mediators in the present study.

Oscillatory tissue mechanics were also addressed in the present study, in order to avoid the influence of kinetics of surface-active molecule absorption-desorption to the surface film and recruitment-derecruitment [26]. $E$ and $R$ increased 

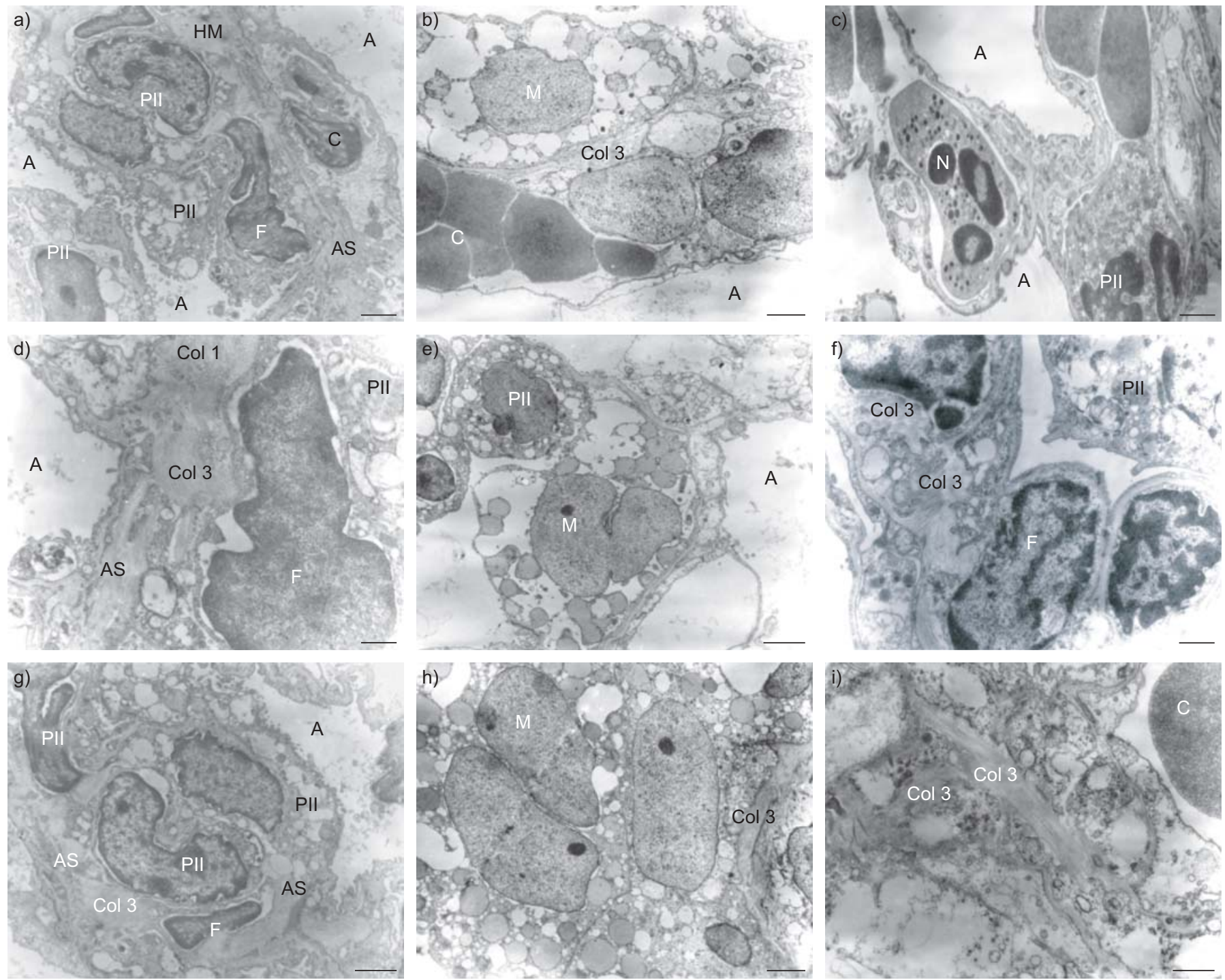

FIGURE 6. Electron microscopy of lung parenchyma after intratracheal instillation of Escherichia coli lipopolysaccharide (10 $\mu \mathrm{g})$ in acute lung injury groups, with methylprednisolone ( $2 \mathrm{mg} \cdot \mathrm{kg}^{-1}$ i.v.) at $6 \mathrm{~h}(\mathrm{a}, \mathrm{d}$ and g), $24 \mathrm{~h}$ ( $\mathrm{b}, \mathrm{e}$ and $\mathrm{h}$ ) or daily (for 7 days, beginning at day $1 ; \mathrm{c}, \mathrm{f}$ and $\mathrm{i})$. All groups were analysed at 1 week (a-c), 3 weeks $(\mathrm{d}-\mathrm{f})$ and 8 weeks $(\mathrm{g}-\mathrm{i})$. Note the integrity of the alveolar capillary membrane, and the presence of mainly type III collagen fibres (Col3) after methylprednisolone treatment. HM: hyaline membrane; A: alveolar space; PII: type II pneumocytes; C: capillary; F: fibroblast; AS: alveolar septa; M: macrophage; N: neutrophil; Col1: type I collagen. a) Scale bar $=1,000 \mathrm{~nm}$; b, c, e, f and h) scale bars $=500 \mathrm{~nm}$; d, g and i) scale bars $=250 \mathrm{~nm}$.

significantly in ALI at 1 week and remained elevated until 8 weeks (fig. 2), but $\eta$ did not change. This suggests that the viscoelasticity-related pressure drop at 8 weeks is more likely due to heterogeneity and alveolar collapse (table 1). In previous studies, it was also observed that parenchymal mechanical dysfunction plays an important role in ALI pathophysiology $[8,10]$. These changes in tissue mechanics were accompanied by deposition of collagen and elastic fibres in the alveolar septa (fig. 4). Notably, corticosteroid prevented the in vitro mechanical changes, independent of treatment design (fig. 4).

It was observed in the present study that the fractional area of alveolar collapse was significantly higher in methylprednisolone-treated groups $(\sim 19 \%)$ compared with control $(6 \%)$, but Est,L was near to normal at 8 weeks.
Additionally, in nontreated ALI lungs, collagen and elastic fibre content was nearly doubled compared with control, but no significant changes were observed in Est,L. These apparent discrepancies could be attributed to the occasional dissociation between lung histology and mechanics. In fact, a threshold of alveolar collapse and collagen fibre content modifications to induce changes in $E s t, \mathrm{~L}$ has been reported [8, 27]. In contrast, in vitro lung mechanical changes were usually very well correlated with modifications in the amount of collagen and elastic fibres $[8,10]$.

MMPs are zinc-dependent endopeptidases, known for their ability to cleave one or several constituents of the ECM [28]. MMP-2 is preferentially secreted from fibroblasts and various epithelial cells including airway epithelial cells, while MMP-9 is preferentially expressed by inflammatory cells [28]. In 
TABLE 2 Semiquantitative analysis of electron microscopy

\begin{tabular}{|c|c|c|c|c|c|}
\hline Analysis group & $\begin{array}{c}\text { Alveolar capillary } \\
\text { barrier }\end{array}$ & Type II epithelial cell & Type I collagen fibre & $\begin{array}{c}\text { Type III collagen } \\
\text { fibre }\end{array}$ & Endothelial cell \\
\hline \multicolumn{6}{|l|}{1 week } \\
\hline Control & $0(0-1)$ & $0(0-1)$ & $0(0-1)$ & $1(1-2)$ & $0(0-1)$ \\
\hline ALI+saline & $3(2-4)^{*}$ & $3(2-4)^{*}$ & $1(1-2)^{*}$ & $3(3-4)^{*}$ & $1(1-2)^{*}$ \\
\hline M-6h & $1(0-2)$ & $1(0-1)$ & $1(0-1)$ & $1(1-2)$ & $0(0-1)$ \\
\hline \multicolumn{6}{|l|}{3 weeks } \\
\hline Control & $0(0-1)$ & $0(0-1)$ & $0(0-1)$ & $1(1-2)$ & $0(0-1)$ \\
\hline ALI+saline & $2(1-3)^{*}$ & $3(1-3)^{*}$ & $3(2-3)^{*, \#}$ & $2(2-3)^{*}$ & $1(1-1)^{*}$ \\
\hline M-6h & $0(0-1)$ & $1(0-1)$ & $1(0-2)$ & $1(1-2)$ & $0(0-1)$ \\
\hline$M-24 h$ & $0(0-1)$ & $0(0-1)$ & $1(0-1)$ & $1(1-2)$ & $0(0-1)$ \\
\hline$M-7 D$ & $1(0-1)$ & $0(0-1)$ & $1(0-1)$ & $1(1-2)$ & $0(0-1)$ \\
\hline M-6h & $1(0-1)$ & $0(0-1)$ & $1(0-1)$ & $1(1-2)$ & $0(0-1)$ \\
\hline$M-24 h$ & $1(0-2)$ & $0(0-1)$ & $1(0-2)$ & $1(1-2)$ & $0(0-1)$ \\
\hline$M-7 D$ & $1(0-1)$ & $1(0-1)$ & $1(0-2)$ & $1(0-2)$ & $0(0-1)$ \\
\hline
\end{tabular}

Data are presented as median (range) lung tissue scores, with five mice in each group. Lung tissue scoring was performed independently by two different investigators The pathological findings were graded according to a 5-point semiquantitative severity-based scoring system: 0) normal lung parenchyma; 1) changes in 1-25\% of the examined tissue; 2) changes in $26-50 \%$ of the examined tissue; 3) changes in $51-75 \%$ of the examined tissue; and 4) changes in $76-100 \%$ of the examined tissue. In the control group, saline was intratracheally instilled $(0.05 \mathrm{~mL}$ ), and the acute lung injury (ALI) mice received Escherichia coli lipopolysaccharide (10 $\mu \mathrm{g})$. The ALI groups were

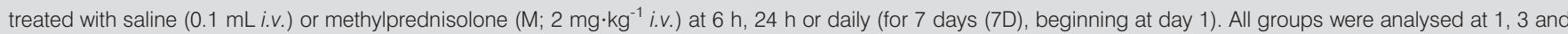
8 weeks. *: $p<0.05$ compared with control; ${ }^{*}: p<0.05$ compared with ALI+saline at 1 week; $": p<0.05$ compared with ALI+saline at 3 weeks.

particular, gelatinases such as MMP-2 and MMP-9 play a crucial role in remodelling of the basement membrane in various lung diseases because these enzymes are capable of degrading type IV collagen. In the present study, macrophages expressed more MMP-9 than neutrophils at 1, 3 and 8 weeks. Neutrophil and macrophage expression of MMP-9 was more prominent at 1 week but fell with the time course of lung injury (fig. 6). MMP-2 presented a weak expression in macrophages compared with MMP-9 (fig. 7), although it was higher in ALI than in the controls. In agreement with the current results, CORBEL et al. [29] observed early induction of MMP-9 and MMP-2 in a murine model of LPS-induced ALI. Corticosteroid therapy decreased the expression of MMP-9 and MMP-2 independent of treatment design, in accordance with
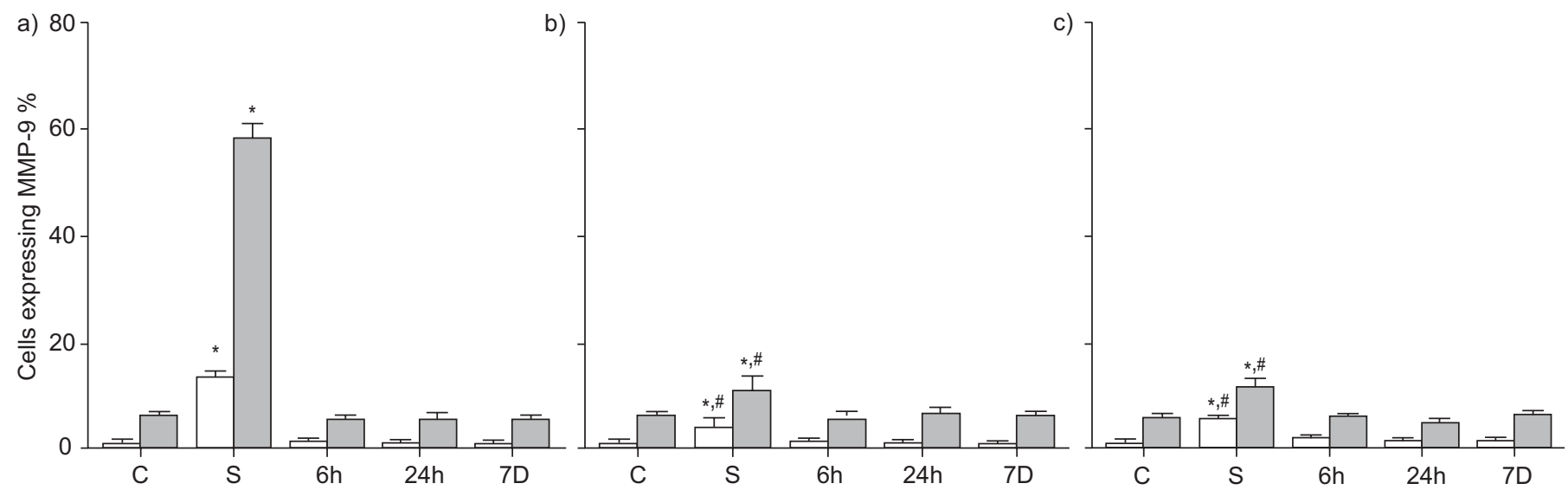

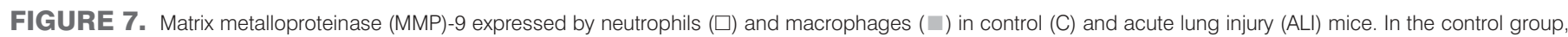

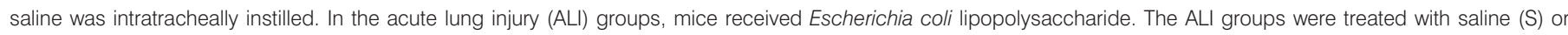

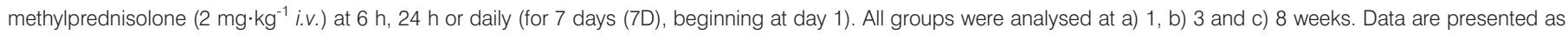
mean \pm SEM. * $: p<0.05$ compared with control; ${ }^{*}: p<0.05$ compared with $S$ at 1 week. 

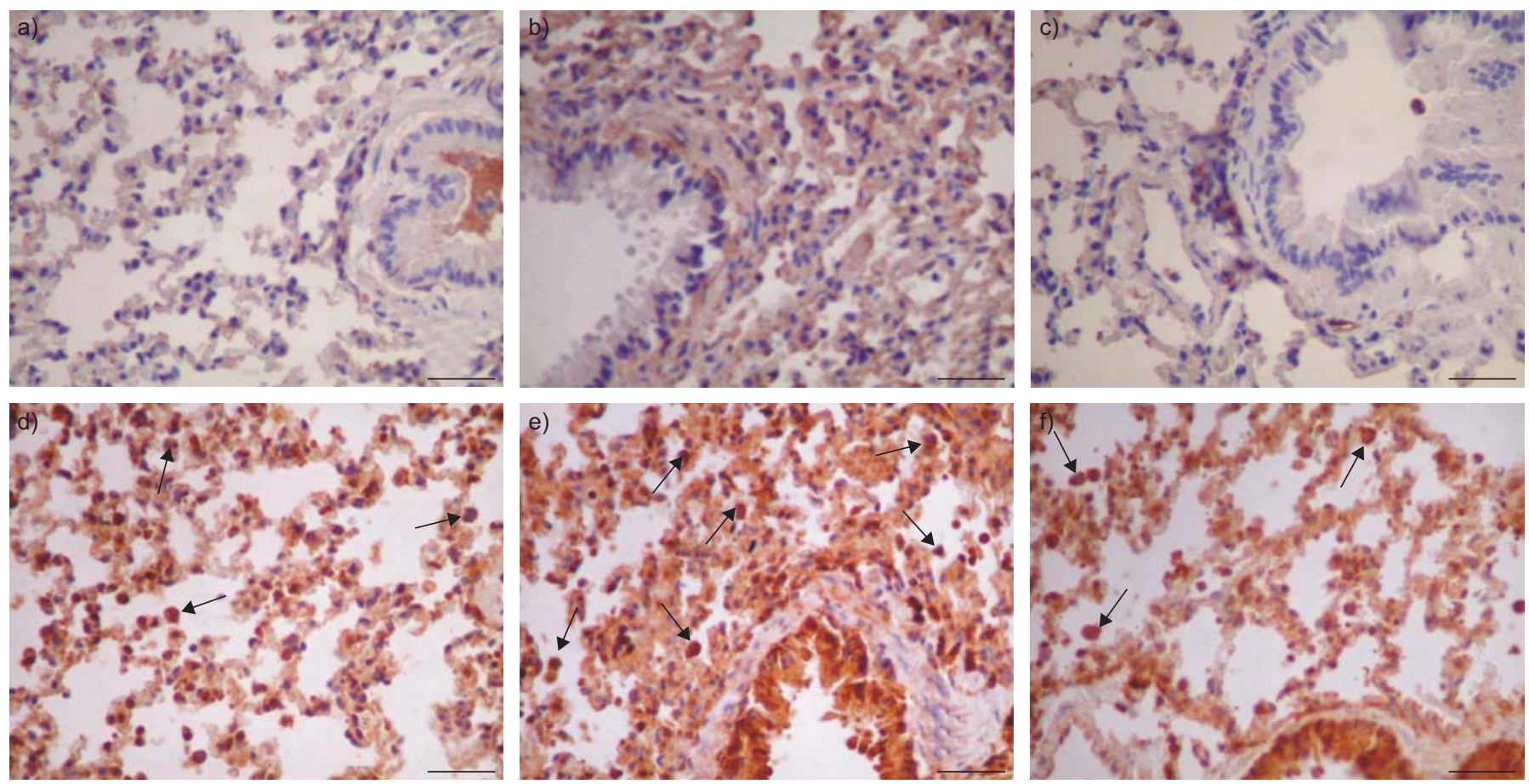

FIGURE 8. Immunohistochemical staining detection at 1 week of matrix metalloproteinase (MMP)-2 (a-c) and -9 (d-f) in control (a and d) and acute lung injury groups treated with saline ( $\mathrm{b}$ and e) and methylprednisolone at $6 \mathrm{~h}$ (c and f). Since no significant differences were found among the three treatments, only the methylprednisolone at $6 \mathrm{~h}$ group is presented. Note the weak MMP-2 expression in alveolar macrophages and epithelial cells. MMP-9 was strongly expressed in alveolar macrophages (arrows). Original magnification: $200 \times$. Scale bars $=10 \mu \mathrm{m}$.

TABLE 3 Matrix metalloproteinase-2 in lung tissue

\begin{tabular}{lcc} 
Analysis group & Epithelial cells & Macrophages \\
\hline 1 week & & \\
Control & $1.08 \pm 0.22$ & $0.71 \pm 0.22$ \\
ALI+saline & $3.55 \pm 0.31^{*}$ & $3.45 \pm 0.55^{\star}$ \\
M-6h & $1.10 \pm 0.43$ & $0.74 \pm 0.43$ \\
M-24h & $0.98 \pm 0.55$ & $0.81 \pm 0.32$ \\
M-7D & $1.07 \pm 0.52$ & $0.75 \pm 0.23$ \\
3 weeks & & \\
Control & $1.12 \pm 0.38$ & $0.73 \pm 0.24$ \\
ALI+saline & $2.46 \pm 0.31^{*, \#}$ & $2.03 \pm 0.26^{*, \#}$ \\
M-6h & $0.98 \pm 0.25$ & $0.74 \pm 0.43$ \\
M-24h & $0.87 \pm 0.32$ & $0.81 \pm 0.32$ \\
M-7D & $0.82 \pm 0.21$ & $0.75 \pm 0.23$ \\
8 weeks & & \\
Control & $1.31 \pm 0.43$ & $0.53 \pm 0.17$ \\
ALI+saline & $2.02 \pm 0.57^{*, \#}$ & $1.52 \pm 0.98^{*, \#}$ \\
M-6h & $0.81 \pm 0.35$ & $0.84 \pm 0.33$ \\
M-24h & $0.97 \pm 0.22$ & $0.71 \pm 0.42$ \\
M-7D & $0.92 \pm 0.31$ & $0.72 \pm 0.22$ \\
\hline
\end{tabular}

Data are presented as mean \pm SEM \%. In the control group, saline was intratracheally instilled $(0.05 \mathrm{~mL})$, and the acute lung injury (ALI) mice received Escherichia coli lipopolysaccharide $(10 \mu \mathrm{g})$. The ALI groups were treated with saline ( $0.1 \mathrm{~mL}$ i.V.) or methylprednisolone ( $\mathrm{M} ; 2 \mathrm{mg} \cdot \mathrm{kg}^{-1} \mathrm{i} . \mathrm{V}$.) at $6 \mathrm{~h}, 24 \mathrm{~h}$ or daily (for 7 days (7D), beginning at day 1). All groups were analysed at 1, 3 and 8 weeks. ${ }^{*}: p<0.05$ compared with control; ${ }^{*}: p<0.05$ compared with ALI+saline at 1 week previous studies [30, 31]. In this respect, corticosteroids cause inhibition of the transcriptional activity of several different genes involved in connective tissue remodelling [32], avoiding fibroelastogenesis. Methylprednisolone may also act in a pretranslational level on alveolar macrophage metalloproteinases, as shown by decreased steady-state mRNA for collagenase after addition of steroid to LPS-stimulated cells [33].

The present study has some limitations that need to be addressed. 1) A specific experimental ALI model induced by i.t. LPS administration was used. Thus, it is not known whether the results can be directly applied to other experimental models of ALI. A model of pulmonary ALI was used, since, in a previous study [15], it had been observed that methylprednisolone was effective in inhibiting fibrogenesis independent of the aetiology of ALI, but its ability to attenuate inflammatory responses and lung mechanical changes was more beneficial in pulmonary compared with extrapulmonary ALI. 2) The degree of ALI induced by i.t. administration of LPS was moderate. However, a significant lung injury was achieved, as demonstrated by changes in lung mechanics, an increased amount of atelectasis, inflammatory cells in the lung and enhanced remodelling processes, associated with high mortality. In addition, the animals breathed spontaneously, thus possible side-effects induced by prolonged mechanical ventilation were avoided. 3) Only i.v. methylprednisolone was used; therefore, the possibility cannot be excluded that other corticosteroids and/or modes of delivery may lead to different results. 4) Inflammatory cytokines were not measured early in the course of ALI. 5) In clinical practice it is difficult to recognise the exact moment of ALI/ARDS onset, thus the exact 
moment when corticosteroid should be administered is difficult to determine. 6) Finally, the current results cannot be directly transferred to clinical practice, but require further investigations. However, the current authors believe that the present data may improve knowledge regarding the appropriate use of corticosteroids in mild ALI.

In conclusion, the present model of acute lung injury induced by intratracheal instillation of Escherichia coli lipopolysaccharide markedly affected not only lung mechanical properties, but also triggered the influx of inflammatory cells in the lung parenchyma and fibroelastosis that did not normalise even 8 weeks after injury. Methylprednisolone led to a complete resolution of in vivo and in vitro lung mechanical and histological alterations and avoided fibroelastogenesis, independent of the corticosteroid treatment design. The current authors suggest that early short-term, low-dose methylprednisolone treatment may be effective as prolonged therapy in acute lung injury.

\section{ACKNOWLEDGEMENTS}

The authors would like to express gratitude to the following people: A. Benedito da Silva (Laboratory of Pulmonary Investigation, Carlos Chagas Filho Biophysics Institute, Federal University of Rio de Janeiro, Rio de Janeiro, Brazil) for animal care; M.R. Taborda Simone (Dept of Pathology, Faculty of Medicine, University of São Paulo, São Paulo, Brazil) and A.L. Neves da Silva (Laboratory of Pulmonary Investigation, Carlos Chagas Filho Biophysics Institute) for help with microscopy; J. Lima do Nascimento (Laboratory of Pulmonary Investigation, Carlos Chagas Filho Biophysics Institute) for her skilful technical assistance during the experiments; and M.E. Schöttler (Rio de Janeiro) for assistance in editing the manuscript.

\section{REFERENCES}

1 Meduri GU, Marik PE, Chrousos GP, et al. Steroid treatment in ARDS: a critical appraisal of the ARDS network trial and the recent literature. Intensive Care Med 2008; 34: 61-69.

2 Luce JM, Montgomery AB, Marks JD, Turner J, Metz CA, Murray JF. Ineffectiveness of high-dose methylprednisolone in preventing parenchymal lung injury and improving mortality in patients with septic shock. Am Rev Respir Dis 1988; 138: 62-68.

3 Bernard GR, Luce JM, Sprung CL, et al. High-dose corticosteroids in patients with the adult respiratory distress syndrome. N Engl J Med 1987; 317: 1565-1570.

4 Meduri GU, Headley AS, Golden E, et al. Effect of prolonged methylprednisolone therapy in unresolving acute respiratory distress syndrome: a randomized controlled trial. JAMA 1998; 280: 159-165.

5 Annane D, Sébille V, Bellissant E, Ger-Inf-05 Study Group, Effect of low doses of corticosteroids in septic shock patients with or without early acute respiratory distress syndrome. Crit Care Med 2006; 34: 22-30.

6 Lee HS, Lee JM, Kim MS, Kim HY, Hwangbo B, Zo JI. Lowdose steroid therapy at an early phase of postoperative acute respiratory distress syndrome. Ann Thorac Surg 2005; 79: 405-410.
7 Meduri GU, Golden E, Freire AX, et al. Methylprednisolone infusion in early severe ARDS: results of a randomized controlled trial. Chest 2007; 131: 954-963.

8 Rocco PRM, Souza AB, Faffe DS, et al. Effect of corticosteroid on lung parenchyma remodeling at an early phase of acute lung injury. Am J Respir Crit Care Med 2003; 168: 677-684.

9 Bates JH, Rossi A, Milic-Emili J. Analysis of the behavior of the respiratory system with constant inspiratory flow. J Appl Physiol 1985; 58: 1840-1848.

10 Rocco PRM, Negri EM, Kurtz PM, et al. Lung tissue mechanics and extracellular matrix remodeling in acute lung injury. Am J Respir Crit Care Med 2001; 164: 1067-1071.

11 Fredberg JJ, Stamenovic D. On the imperfect elasticity of lung tissue. J Appl Physiol 1989; 67: 2408-2419.

12 Weibel ER. Morphometry: stereological theory and practical methods. In: Gil J, ed. Models of Lung Disease Microscopy and Structural Methods. New York, Marcel Dekker, 1990; pp. 199-247.

13 Santos FB, Nagato LK, Boechem NM, et al. Time course of lung parenchyma remodeling in pulmonary and extrapulmonary acute lung injury. J Appl Physiol 2006; 100: 98-106.

14 Menezes SL, Bozza PT, Neto HC, et al. Pulmonary and extrapulmonary acute lung injury: inflammatory and ultrastructural analyses. J Appl Physiol 2005; 98: 1777-1783.

15 Leite-Junior JH, Garcia CS, Souza-Fernandes AB, et al. Methylprednisolone improves lung mechanics and reduces the inflammatory response in pulmonary but not in extrapulmonary mild acute lung injury in mice. Crit Care Med 2008; 36: 2621-2628.

16 Meduri GU, Muthiah MP, Carratu P, Eltorky M, Chrousos GP. Nuclear factor- $\mathrm{B}$ - and glucocorticoid receptor- $\alpha$-mediated mechanisms in the regulation of systemic and pulmonary inflammation during sepsis and acute respiratory distress syndrome. Evidence for inflammation-induced target tissue resistance to glucocorticoids. Neuroimmunomodulation 2005; 12: 321-338.

17 Rhen T, Cidlowski JA. Antiinflammatory action of glucocorticoids - new mechanisms for old drugs. N Engl J Med 2005; 353: 1711-1723.

18 Esbenshade AM, Newman JH, Lams PM, Jolles H, Brigham KL. Respiratory failure after endotoxin infusion in sheep: lung mechanics and lung fluid balance. J Appl Physiol 1982; 53: 967-976.

19 Steinberg KP, Hudson LD, Goodman RB, et al. Efficacy and safety of corticosteroids for persistent acute respiratory distress syndrome. N Engl J Med 2006; 354: 1671-1684.

20 Greos LS, Vichyanond P, Bloedow DC, et al. Methylprednisolone achieves greater concentrations in the lung than prednisolone. A pharmacokinetic analysis. Am Rev Respir Dis 1991; 144: 586-592.

21 Keh D, Sprung CL. Use of corticosteroid therapy in patients with sepsis and septic shock: an evidence-based review. Crit Care Med 2004; 32: Suppl. 11, S527-S533.

22 Confalonieri M, Urbino R, Potena A, et al. Hydrocortisone infusion for severe community-acquired pneumonia: a preliminary randomized study. Am J Respir Crit Care Med 2005; 171: 242-248.

23 Marshall RP, Bellingan G, Webb S, et al. Fibroproliferation occurs early in the acute respiratory distress syndrome and impacts on outcome. Am J Respir Crit Care Med 2000; 162: 1783-1788. 
24 De Jonghe B, Sharshar T, Lefaucheur JP, et al. Paresis acquired in the intensive care unit: a prospective multicenter study. JAMA 2002; 288: 2859-2867.

25 Braude S, Haslam P, Hughes D, MacNaughton P, Evans TW. Chronic adult respiratory distress syndrome a role for corticosteroids? Crit Care Med 1992; 20: 1187-1189.

26 Suki B, Ito S, Stamenovic D, Lutchen KR, Ingenito EP. Biomechanics of the lung parenchyma: critical roles of collagen and mechanical forces. J Appl Physiol 2005; 98: 1892-1899.

27 Contador RS, Chagas PS, Vasconcellos FP, et al. Evaluation of respiratory mechanics and lung histology in a model of atelectasis. Respir Physiol Neurobiol 2003; 137: 61-68.

28 Johnson LL, Dyer R, Hupe DJ. Matrix metalloproteinases. Curr Opin Chem Biol 1998; 2: 466-471.

29 Corbel M, Theret N, Caulet-Maugendre S, et al. Repeated endotoxin exposure induces interstitial fibrosis associated with enhanced gelatinase (MMP-2 and MMP-9) activity. Inflamm Res 2001; 50: 129-135.
30 Corbel M, Lagente V, Théret N, Germain N, Clément B, Boichot E. Comparative effects of betamethasone, cyclosporin and nedocromil sodium in acute pulmonary inflammation and metalloproteinase activities in bronchoalveolar lavage fluid from mice exposed to lipopolysaccharide. Pulm Pharmacol Ther 1999; 12: 165-171.

31 Carver JE, Galloway WA, Robinson C. Inhibition of gelatinase activity in human airway epithelial cells and fibroblasts by dexamethasone and beclomethasone. $\mathrm{Br} \mathrm{J}$ Pharmacol 1999; 127: 1119-1128.

32 Tomic R, Lassiter CC, Ritzenthaler JD, Rivera HN, Roman J. Anti-tissue remodeling effects of corticosteroids: fluticasone propionate inhibits fibronectin expression in fibroblasts. Chest 2005; 127: 257-265.

33 Shapiro SD, Campbell EJ, Kobayashi DK, Welgus HG. Dexamethasone selectively modulates basal and lipopolysaccharide-induced metalloproteinase and tissue inhibitor of metalloproteinase production by human alveolar macrophages. J Immunol 1991; 146: 2724-2729. 